\section{Adaptação transcultural e investigação psicométrica da Escala de Estresse na Alimentação de Salzburg (SSES) para uma amostra de adultos brasileiros}

\section{Cross-cultural adaptation and psychometric investigation of the Salzburg Stress Eating Scale (SSES) in a sample of Brazilian adults}

\author{
Adaptación transcultural e investigación \\ psicométrica de la Escala de Estrés en la \\ Alimentación de Salzburg (SSES) para \\ una muestra de adultos brasileños
}

Priscila Carvalho Santos 1 Wanderson Roberto da Silva 1 João Marôco 2 Juliana Alvares Duarte Bonini Campos 1

\section{Resumo}

Os objetivos deste estudo foram adaptar a Escala de Estresse na Alimentação de Salzburg (SSES) para o português brasileiro, estimar suas propriedades psicométricas para uma amostra de adultos jovens e verificar, separadamente para cada sexo, a relação da idade e do indice de massa corporal (IMC) com a forma de lidar com a alimentação frente ao estresse. A adaptação da SSES para o português foi realizada seguindo protocolo consolidado. As propriedades psicométricas foram estimadas para cada sexo a partir das validades fatorial e convergente e da confiabilidade. A invariância foi testada em subamostras independentes para cada sexo. A relação da idade e do IMC com a alimentação frente ao estresse foi investigada para cada sexo usando a modelagem de equações estruturais. A prevalência dos indivíduos em categorias que representaram manutenção, redução ou aumento da alimentação frente ao estresse foi calculada. Participaram do estudo 1.030 individuos (61,8\% mulheres) com média de idade de 25,5 (DP = 5,3) anos. O modelo original da SSES apresentou bom ajuste para a amostra feminina, mas, para a masculina, um item foi excluído, e uma correlação residual foi inserida. Esses modelos foram invariantes em subamostras independentes. O IMC elevado influenciou na alimentação frente ao estresse. Os homens mantêm a alimentação habitual, enquanto as mulheres a aumentam frente ao estresse. A versão em português da SSES será útil para investigar a alimentação frente ao estresse no Brasil. Modelos diferentes da SESS foram ajustados para cada sexo. O IMC foi uma variável significativa para avaliar a alimentação frente ao estresse.

\author{
Correspondência \\ J. A. D. B. Campos \\ Universidade Estadual Paulista Júlio de Mesquita Filho. \\ Rod. Araraquara-Jaú, Km 1, Araraquara, SP \\ 14800-903, Brasil. \\ juliana.campos@unesp.br \\ 1 Universidade Estadual Paulista Júlio de Mesquita Filho, \\ Araraquara, Brasil. \\ 2 Instituto Superior de Psicologia Aplicada, Lisboa, Portugal.
}




\section{Introdução}

O estresse pode ser definido como uma reação não específica do corpo, em resposta a demandas ambientais, sociais ou internas 1,2, que coloca o organismo em estado de alerta e pode provocar alterações físicas e emocionais. Essa condição pode ser desencadeada por diferentes razões e gerar respostas que variam individualmente 3, e, por esse motivo, os resultados podem ser distintos 2,3,4,5,6. Diante de um estresse agudo, respostas adaptativas são ativadas buscando o restabelecimento do equilíbrio orgânico. Contudo, frente à não existência ou ao esgotamento de recursos para enfrentamento do estresse, esse pode se prolongar e se tornar crônico, dificultando o domínio da situação ${ }^{3}$. Nesse sentido, alguns indivíduos podem apresentar respostas desadaptativas diante de situações estressoras e utilizar recursos como, por exemplo, a alimentação para lidar com o estressor ${ }^{7}$. Assim, uma das consequências que o estresse pode causar na vida das pessoas é a mudança do comportamento alimentar. Explicações distintas justificam essa alteração como, por exemplo, fatores fisiológicos e psicológicos 2 .

Com relação aos fatores fisiológicos, uma cascata de acontecimentos internos, que inclui a liberação de substâncias como hormônios e neurotransmissores, é responsável por influenciar a ingestão alimentar e a regulação metabólica. No que se refere aos fatores psicológicos, pode-se mencionar situações de aprendizagem, enfrentamento e mecanismos de regulação de emoções 2. Além disso, o impacto do estresse no comportamento alimentar dos indivíduos pode depender da intensidade, duração, fonte ou tipo de estímulo estressor 8,9. Ainda, características situacionais e individuais também devem ser consideradas 9,10. Assim, a multidimensionalidade desse processo certamente pode justificar o fato de os resultados encontrados na literatura referentes ao comportamento alimentar frente ao estresse serem tão distintos.

As mudanças no comportamento alimentar que ocorrem devido ao estresse podem ser observadas, especialmente, em relação à escolha dos alimentos, ingestão alimentar e apetite 1,11. A literatura aponta que a maior parte dos indivíduos tende a aumentar ou reduzir sua ingestão alimentar, enquanto que pequena parcela mantém sua alimentação inalterada diante de situações estressoras 1,12. Com relação aos tipos de alimentos selecionados, a literatura parece consensual ao apontar o aumento do interesse voltado aos alimentos palatáveis como, por exemplo, doces 5,12. Reichenberger et al. 2 destacam que, em momentos de estresse, as pessoas que costumam restringir a alimentação aumentam a ingestão de alimentos em comparação com as que não apresentam tal comportamento. Outro ponto a ser destacado é que, durante episódios de alimentação excessiva, motivados por emoções e/ ou estresse, pode ocorrer um alívio ou entorpecimento das sensações desagradáveis que motivaram a alimentação. Assim, os indivíduos podem experienciar um ligeiro aumento de ansiedade devido ao sentimento de culpa por comer demais, o que pode produzir efeitos desfavoráveis para a saúde física e mental 13. Portanto, destaca-se a importância de avaliar a alimentação frente ao estresse visando desenvolver intervenções precoces em prol da saúde da população.

A avaliação da alimentação relacionada ao estresse pode ser realizada por meio de instrumentos psicométricos como, por exemplo, o Questionário de Avaliação da Alimentação devido às Emoções e Estresse (Eating and Appraisal due to Emotions and Stress Questionnaire - EADES) 14, o Questionário Emocional do Apetite (Emotional Appetite Questionnaire - EMAQ) 15 e a Escala de Estresse na Alimentação de Salzburg (Salzburg Stress Eating Scale - SSES) 10. Entre esses, a SSES 10 é o único instrumento que pode ser particularmente relevante para a identificação do estresse genuíno na alimentação, uma vez que não inclui nenhum outro estado ou emoção além do estresse. A SSES foi desenvolvida em 2018, nas línguas inglesa e alemã 10 . Além dessas, uma versão em holandês e outra em árabe estão disponíveis em ambiente virtual de livre acesso 16 . Na língua portuguesa, até o presente momento, nenhuma versão da SSES foi apresentada. Esse instrumento poderá ser útil no país tanto para o levantamento de informações direcionadas ao cuidado com a alimentação, de modo a contribuir para a formulação de medidas de prevenção e intervenção, quanto para comparação de dados advindos de outras populações, ampliando as discussões sobre o tema. Contudo, quando do uso de instrumentos como a SSES, a avaliação das propriedades psicométricas torna-se imprescindível, visando garantir a validade e a confiabilidade dos dados obtidos 17.

Quando da investigação da alimentação relacionada ao estresse, a literatura 4,10,18 tem apontado diferenças entre mulheres e homens. Jiang et al. 18 sugerem que as mulheres tendem a utilizar a alimentação para lidar com algumas situações da vida, diferente dos homens que recorrem a outros métodos, 
como uso de álcool e tabaco. Nesse sentido, avaliar o comportamento alimentar frente ao estresse separadamente para cada sexo é importante para auxiliar na elaboração de estratégias preventivas/ intervencionistas mais assertivas. Outras características como, por exemplo, índice de massa corporal (IMC) e idade também podem interferir na alimentação. O estudo realizado por Greeno et al. 19 aponta que os indivíduos do sexo feminino e aqueles classificados segundo o IMC com sobrepeso ou obesidade apresentam maior ingestão de alimentos durante situações de estresse. Quanto à idade, alguns trabalhos 20,21 relatam que os mais jovens costumam apresentar maior desejo de se alimentar diante de situações emocionais negativas. Portanto, investigar a relação dessas características com a alimentação frente ao estresse pode contribuir para a elaboração de protocolos mais direcionados.

Diante do exposto, os objetivos do presente estudo foram adaptar a SSES para o português brasileiro, estimar suas propriedades psicométricas para uma amostra de adultos jovens e verificar, separadamente para cada sexo, a relação da idade e do IMC com a forma de lidar com a alimentação frente ao estresse.

\section{Métodos}

\section{Desenho de estudo e delineamento amostral}

Trata-se de estudo observacional do tipo transversal com delineamento amostral não probabilístico. Para garantir o adequado poder das análises estatísticas, de modo a sustentar a inferência dos resultados, o tamanho mínimo da amostra foi calculado com base no número de itens do modelo original da SSES $(\mathrm{k}=10)$. Considerando a necessidade de, pelo menos, 15 respondentes para cada item da escala 22 e uma possível taxa de perda de 10\%, o tamanho amostral mínimo foi estimado em 167 participantes. Contudo, considerando a realização da análise de invariância fatorial, o tamanho amostral mínimo foi duplicado, ou seja, durante o trabalho de campo, foi fixada a meta de coletar, pelo menos, 334 questionários de cada sexo, uma vez que as análises estatísticas foram realizadas separadamente para as amostras feminina e masculina. Com essa estratégia, o tamanho amostral estimado foi considerado suficiente para realizar todas as análises estatísticas.

\section{Participantes}

Estudantes, funcionários técnico-administrativos e docentes de uma universidade pública do Estado de São Paulo, no Brasil, foram convidados para participar do estudo por meio de e-mails, redes sociais e convites pessoais. Como critérios de inclusão, considerou-se ter idade entre 18 e 35 anos e ser capaz de ler e responder sozinho as perguntas da pesquisa. Já como critérios de exclusão, adotouse estar gestante ou lactante e ter deficiência visual total. Os indivíduos que demonstraram interesse procuraram os pesquisadores responsáveis e receberam formalmente os devidos esclarecimentos sobre o estudo. Aqueles que concordaram, voluntariamente, em participar da pesquisa preencheram, em papel, a versão em português da SSES e um questionário de caracterização da amostra em uma sala reservada da universidade. Após finalizar o preenchimento, cada participante foi orientado a indicar novos indivíduos para participar da pesquisa. Portanto, adotou-se amostragem em bola de neve. Quando o tamanho amostral mínimo foi alcançado para ambos os sexos, a coleta de dados foi interrompida. Como a participação de indivíduos do sexo feminino é, geralmente, mais comum em pesquisas voluntárias, o tamanho da amostra dessa população foi maior no presente estudo, pois, até alcançar o tamanho mínimo da amostra masculina, optou-se por não interromper a recolha de dados femininos.

As informações autorrelatadas pelos participantes para caracterização da amostra foram sexo, idade, peso corporal (kg), altura (m) e nível econômico. O cálculo do IMC e a classificação do estado nutricional antropométrico foram realizados conforme diretrizes da Organização Mundial da Saúde (OMS) 23,24 Para estimar o nível econômico, foi utilizado o Critério de Classificação Econômica Brasil 2020 25, e os participantes foram distribuídos em classes conforme a renda média mensal bruta estimada da família (classes: $\mathrm{A}=\mathrm{R} \$ 25.554,33 ; \mathrm{B}=\mathrm{R} \$$ 8.460,39; $\mathrm{C}=\mathrm{R} \$ 2.417,04 ; \mathrm{D}$ e $\mathrm{E}=\mathrm{R} \$ 719,81$ ). 


\section{Instrumento de medida}

A SSES foi desenvolvida originalmente nas línguas inglesa e alemã 10, com 10 itens e modelo unifatorial para avaliar o estresse na alimentação. Em relação às opções de resposta, essas foram apresentadas em escala do tipo Likert de 5 pontos ( 1 = eu como muito menos do que o habitual, 2 = eu como menos do que o habitual, 3 = eu como tanto quanto o habitual, 4 = eu como mais do que o habitual, $5=\mathrm{eu}$ como muito mais do que o habitual). Pontuações elevadas no instrumento indicam aumento do ato de comer quando o indivíduo se sente estressado.

\section{Adaptação da SSES para o português brasileiro}

Esse processo foi realizado em cinco etapas, nomeadas como: (1) tradução; (2) síntese das traduções; (3) retrotradução da versão sintetizada; (4) avaliação de especialistas da versão obtida e (5) pré-teste na população alvo. Nessas etapas, quatro equivalências foram avaliadas nas seguintes áreas: (I) semântica: a qual refere-se ao significado das palavras em relação ao vocabulário e gramática; (II) idiomática: que se destina a verificar a adequação do significado das expressões; (III) conceitual: para garantir a manutenção do conceito teórico proposto originalmente; e (IV) cultural: visando adaptar, se necessário, ao contexto cultural do público alvo do estudo 26 . As primeiras etapas realizadas foram tradução, síntese e retrotradução, com o objetivo de alcançar as equivalências semântica e idiomática. Para tanto, a versão em inglês da SSES foi enviada para quatro tradutores independentes, nativos do Brasil e com conhecimento avançado do idioma de origem do instrumento. As versões traduzidas foram comparadas pelos autores do presente estudo que elaboraram uma única versão em português. Essa foi enviada para um retrotradutor independente, nativo em inglês e com conhecimento avançado da língua portuguesa. A retrotradução foi comparada com a versão original da SSES em inglês, e constatou-se similaridade de conteúdo. Posteriormente, a etapa de avaliação de especialistas foi realizada para alcançar as equivalências conceitual e cultural. Nessa, a versão em português foi analisada por dois brasileiros especialistas em psicometria e saúde, com experiência na cultura de Portugal, os quais verificaram e atestaram que o conteúdo obtido era teoricamente fiel ao original, assim como adequado para a cultura brasileira. Os especialistas também indicaram que a versão em português obtida pode ser útil para o contexto de Portugal, mas destacaram que, antes de utilizar essa versão na população, é necessário realizar um pré-teste. No presente estudo, a etapa do pré-teste foi realizada apenas com indivíduos brasileiros $(n=32$; mulheres $=71,9 \%$; média de idade $=28,9$ anos e desvio padrão $[\mathrm{DP}]=6,6$ anos) que responderam a versão em português da SSES e discutiram o conteúdo com os pesquisadores. Como nenhum participante relatou dificuldade de preenchimento e/ou compreensão da escala, o conteúdo foi considerado adequado. Apresenta-se, no Quadro 1, a versão em português da SSES.

\section{Análises psicométricas}

Todas as análises foram realizadas separadamente para as amostras feminina e masculina. Primeiramente, a estatística descritiva das respostas dadas aos itens da SSES foi conduzida para verificar a distribuição dos dados. Nessa, os valores absolutos de assimetria e curtose menores do que 3 e 7 , respectivamente, foram considerados adequados para atestar a sensibilidade psicométrica da escala 17.

Para analisar a validade de construto do modelo fatorial original da SSES 10, utilizou-se as validades fatorial e convergente. A validade fatorial foi avaliada por meio de análise fatorial confirmatória (AFC) com o objetivo de verificar a qualidade de ajustamento da estrutura (i.e., conjunto de itens e composição do fator) aos dados. O média de quadrados mínimos ponderados e variância ajustada (weighted least squares mean and variance adjusted - WLSMV) foi utilizado como estimador por ser tratar de um método robusto, que visa obter as estimativas dos parâmetros que melhor reproduzam os dados. A qualidade de ajustamento foi verificada por meio das estimativas observadas nos índices: índice de ajuste comparativo (comparative fit index - CFI), índice de Tucker-Lewis (Tucker-Lewis index - TLI) e raiz quadrada média residual padronizada (standardized root mean square residual - SRMR). O CFI e o TLI são índices relativos que têm como objetivo comparar o modelo com pior e com melhor ajustamento possível. Já o SRMR é um índice absoluto que visa avaliar o modelo per se, sem comparação com outros modelos. O ajustamento foi considerado aceitável quando CFI e TLI $\geq 0,90$ e 


\section{Quadro 1}

Versões em inglês e em português da Escala de Estresse na Alimentação de Salzburg.

\begin{tabular}{|c|c|}
\hline VERSÃO ORIGINAL 10 & VERSÃO EM PORTUGUÊS \\
\hline Salzburg Stress Eating Scale & Escala de Estresse na Alimentação de Salzburg \\
\hline $\begin{array}{l}\text { Response options: } 1=\text { l eat much less than usual, } 2=\text { l eat less } \\
\text { than usual, } 3=\text { I eat just as much as usual, } 4=\text { l eat more than usual, } \\
5=\text { l eat much more than usual }\end{array}$ & $\begin{array}{l}\text { Opções de resposta: } 1 \text { = eu como muito menos do que o habitual, } \\
2 \text { = eu como menos do que o habitual, } 3 \text { = eu como tanto quanto o } \\
\text { habitual, } 4 \text { = eu como mais do que o habitual, } 5 \text { = eu como muito mais } \\
\text { do que o habitual. }\end{array}$ \\
\hline 1. When I am overwhelmed with things I have to do, ... & $\begin{array}{l}\text { 1. Quando eu estou sobrecarregado(a) com coisas que tenho que } \\
\text { fazer, ... }\end{array}$ \\
\hline 2. During periods of great stress, ... & 2. Durante períodos de grande estresse, ... \\
\hline 3. When I feel things are out of control, ... & 3. Quando eu sinto que as coisas estão fora de controle, ... \\
\hline 4. On days where everything seems to go wrong, ... & 4. Nos dias em que tudo parece dar errado, ... \\
\hline 5. While preparing for a strenuous task, ... & 5. Enquanto eu me preparo para fazer uma tarefa árdua/cansativa, ... \\
\hline 6. When I am under pressure, ... & 6. Quando eu estou sob pressão, ... \\
\hline 7. When I feel nervous and stressed, ... & 7. Quando eu me sinto nervoso(a) e estressado(a), ... \\
\hline $\begin{array}{l}\text { 8. When I feel that I have no influence over the important things } \\
\text { in my life, ... }\end{array}$ & $\begin{array}{l}\text { 8. Quando eu sinto que não tenho influência sobre as coisas } \\
\text { importantes da minha vida, ... }\end{array}$ \\
\hline 9. When I feel that I am not really on top of things, ... & 9. Quando eu sinto que não estou realmente no controle das coisas, ... \\
\hline $\begin{array}{l}\text { 10. When I feel difficulties have been piling up so high that I cannot } \\
\text { overcome them,... }\end{array}$ & $\begin{array}{l}\text { 10. Quando eu sinto que as dificuldades se acumulam tanto que eu } \\
\text { não consigo superá-las, ... }\end{array}$ \\
\hline
\end{tabular}

SRMR $<0,08$ 17,27. O peso fatorial $(\lambda)$ de cada item foi considerado adequado quando maior que 0,40. Em caso de não ajustamento do modelo, os índices de modificação maiores que 11, calculados a partir dos multiplicadores de lagrange (ML), foram inspecionados 17.

A validade convergente foi analisada a partir da variância extraída média (VEM), que é calculada com base nos valores dos pesos fatoriais dos itens conforme proposto por Fornell \& Larcker 28 e sustentado por Marôco 17 e Hair Jr. et al. 22. O objetivo dessa análise foi verificar se os itens da SSES representam, de fato, o conceito teórico "estresse na alimentação". Valores $\geq 0,50$ foram indicativos de adequação 28 .

Para verificar a invariância do modelo fatorial ajustado da SSES para cada sexo, realizou-se análise multigrupos. Para tanto, as amostras foram subdivididas aleatoriamente em duas partes iguais (feminina: amostra teste $n=317$ vs. amostra validação $n=317$; masculina: amostra teste $n=198$ vs. amostra validação $n=198$ ). Essa análise foi realizada para verificar a validade externa do modelo fatorial ajustado 29 . O teste foi realizado a partir da diferença de CFI $(\Delta \mathrm{CFI})$, sendo que os valores desse índice, para os modelos configural, métrico e escalar, foram comparados dois a dois. Atestou-se invariância forte quando a redução dos valores de CFI foi menor que -0,0130.

A confiabilidade também foi avaliada para verificar até que ponto o conjunto de itens da SSES pode ser considerado consistente com o que se destina a medir. Para tanto, foi utilizado o coeficiente alfa ordinal $(\alpha)$, sendo considerados adequados valores $\geq 0,7022$.

\section{Escore da SSES}

O escore médio da SSES foi calculado para cada indivíduo considerando as respostas dadas ao conjunto de itens do modelo fatorial ajustado para cada sexo. Como não foi encontrada na literatura uma forma de classificar os indivíduos de acordo com o escore obtido na SSES, foram desenvolvidas, no presente estudo, categorias baseadas nas opções de resposta da escala tipo Likert. Primeiro, definiu-se que o escore médio igual a 3 representaria a categoria "manutenção da alimentação habitual frente 
ao estresse", uma vez que esse valor é o ponto médio da escala tipo Likert e que, de acordo com a instrução do instrumento, refere-se a "comer tanto quanto o habitual". Na sequência, definiu-se outras duas categorias, sendo que os participantes que obtiveram escore médio entre 1 e 3 foram alocados na categoria "redução da alimentação habitual frente ao estresse", e aqueles com escore médio entre 3 e 5 foram alocados na categoria "aumento da alimentação habitual frente ao estresse”. Desse modo, três categorias foram formuladas de maneira arbitrária, não envolvendo procedimento estatístico. Portanto, futuros protocolos devem avaliar se o uso desse método é aplicável em outros contextos/amostras. Na sequência, a prevalência de indivíduos em cada categoria foi calculada por ponto e intervalo de 95\% de confiança (IC95\%) para cada sexo e comparadas.

\section{Modelo estrutural}

Após ajustamento do modelo fatorial da SSES para cada sexo, a influência da idade e do IMC (variáveis independentes) na alimentação frente ao estresse (variável dependente) foi verificada utilizando-se a técnica da modelagem de equações estruturais. Essa busca explicar relações múltiplas e inter-relacionadas entre as variáveis com capacidade de representar conceitos não observados, definindo um modelo teórico e medindo o erro no processo de estimativa 22 . Nessa perspectiva, um modelo estrutural hipoteticamente causal foi construído para cada sexo. As trajetórias ( $\beta$ ) foram analisadas e comparadas aos rácios críticos de z 17. A avaliação da qualidade do ajustamento dos modelos estruturais foi realizada a partir dos valores dos índices CFI, TLI e SRMR 17,27. Adotou-se nível de 5\% de significância.

Para realização das análises, foram utilizados os programas SPSS v. 22 (https://www.ibm.com/) e R (http://www.r-project.org) com os pacotes "lavaan" 31, "sem'Tools" 32 e "psych" 33.

\section{Aspectos éticos}

O presente estudo foi aprovado pelo Comitê de Ética em Pesquisa em Seres Humanos da Faculdade de Ciências Farmacêuticas, Universidade Estadual Paulista Júlio de Mesquita Filho, campus de Araraquara (C.A.A.E.: 11469719.1.0000.5426). Todos os participantes assinaram o Termo de Consentimento Livre e Esclarecido.

\section{Resultados}

Concordaram em participar do estudo 1.047 pessoas, contudo, 17 indivíduos não preencheram todos os itens da SSES e foram excluídos. Assim, 1.030 participantes (mulheres =61,8\%) com média de idade de 25,5 (DP = 5,3) anos foram incluídos nas análises. Mais detalhes referentes à caracterização da amostra encontram-se na Tabela 1. A análise descritiva das respostas dadas aos itens da SSES encontra-se na Tabela 2. Nenhum item apresentou desvios nos valores de assimetria e/ou curtose, confirmando a não violação do pressuposto de normalidade dos dados. Os pesos fatoriais dos itens da SSES também estão apresentados na Tabela 2. Para a amostra feminina, todos os itens apresentaram valores adequados $(\lambda=0,49-0,87)$, enquanto, para amostra masculina $(\lambda=0,26-0,91)$, observou-se baixo peso fatorial no item 5 ( $\lambda=0,26$; conteúdo do item: "preparo para tarefa árdua/cansativa").

$\mathrm{O}$ ajustamento do modelo fatorial da escala aos dados encontra-se na Tabela 3. Observou-se que, para a amostra feminina, os valores dos índices estimados foram bons para o CFI $(0,95)$ e o SRMR $(0,06)$ e aceitável para o TLI $(0,93)$. Já para a masculina, todos os índices apresentaram valores no limite do aceitável (CFI = 0,92; TLI =0,90; SRMR =0,08). Assim, uma investigação visando melhorar o ajuste do modelo foi realizada. Como o item 5 apresentou baixo peso fatorial, ele foi excluído. Além disso, uma forte correlação entre os erros dos itens 8 e 9 foi encontrada $(M L=161)$, e, quando essa foi adicionada, observou-se melhora do ajustamento do modelo aos dados (Tabela 3). Desse modo, o modelo fatorial da SSES para a amostra masculina foi considerado adequado após refinamento. Nas análises de validade convergente e confiabilidade, foram observados adequados valores de VEM (amostras: feminina $=0,60$; masculina $=0,62)$ e $\alpha($ amostras: feminina $=0,94$; masculina $=0,93) \mathrm{em}$ ambas as amostras. No que se refere à invariância fatorial, observou-se que, tanto para a amostra 
Tabela 1

Caracterização da amostra $(\mathrm{n}=1.047)$.

\begin{tabular}{|c|c|c|}
\hline \multirow[t]{2}{*}{ Característica } & \multicolumn{2}{|c|}{ Amostra } \\
\hline & Feminina & Masculina \\
\hline IMC [kg/m²] (média $\pm D P)$ & $24,26 \pm 4,23$ & $25,81 \pm 4,45$ \\
\hline \multicolumn{3}{|c|}{ Classificação do estado nutricional antropométrico (\%) } \\
\hline Baixo peso & 2,5 & 1,8 \\
\hline Peso adequado & 60,4 & 42,6 \\
\hline Sobrepeso & 24,6 & 39,6 \\
\hline Obesidade & 12,5 & 16 \\
\hline \multicolumn{3}{|l|}{ Classe econômica * (\%) } \\
\hline A & 22,9 & 32,5 \\
\hline$B$ & 63,3 & 50,0 \\
\hline $\mathrm{C}$ & 13,3 & 17,5 \\
\hline $\mathrm{D}-\mathrm{E}$ & 0,5 & - \\
\hline
\end{tabular}

DP: desvio padrão; IMC: índice de massa corporal.

* Renda média mensal: $A=R \$ 25.545,33, B=R \$ 8.460,39, C=R \$ 2.417,03, D-E=R \$ 719,81$.

Tabela 2

Análise descritiva das respostas dadas aos itens e pesos fatoriais da Escala de Estresse na Alimentação de Salzburg para as amostras de adultos brasileiros.

\begin{tabular}{|c|c|c|c|c|c|c|c|c|c|c|c|c|c|c|}
\hline \multirow[t]{2}{*}{ Item } & \multicolumn{7}{|c|}{ Amostra feminina } & \multicolumn{7}{|c|}{ Amostra masculina } \\
\hline & Me & Md & Mo & DP & As & $\mathrm{Cu}$ & $\lambda$ & Me & Md & Mo & DP & As & $\mathrm{Cu}$ & $\lambda$ \\
\hline 1 & 2,68 & 2 & 2 & 1,26 & 0,34 & $-1,05$ & 0,71 & 2,61 & 3 & 2 & 1,04 & 0,30 & $-0,47$ & 0,70 \\
\hline 2 & 3,13 & 4 & 4 & 1,35 & $-0,18$ & $-1,31$ & 0,87 & 2,79 & 3 & 2 & 1,15 & 0,05 & $-0,95$ & 0,91 \\
\hline 3 & 3,04 & 3 & 4 & 1,30 & $-0,06$ & $-1,26$ & 0,87 & 2,66 & 3 & 3 & 1,06 & 0,08 & $-0,71$ & 0,89 \\
\hline 4 & 3,00 & 3 & 4 & 1,29 & $-0,07$ & $-1,21$ & 0,86 & 2,72 & 3 & 3 & 1,08 & 0,11 & $-0,59$ & 0,78 \\
\hline 5 & 2,88 & 3 & 3 & 0,93 & $-0,13$ & $-0,06$ & 0,49 & 2,95 & 3 & 3 & 0,84 & $-0,24$ & 0,63 & 0,26 \\
\hline 6 & 2,76 & 3 & 2 & 1,21 & 0,26 & $-0,98$ & 0,75 & 2,71 & 3 & 3 & 0,96 & 0,14 & $-0,32$ & 0,71 \\
\hline 7 & 3,09 & 3 & 4 & 1,29 & $-0,19$ & $-1,21$ & 0,86 & 2,82 & 3 & 3 & 1,07 & 0,08 & $-0,73$ & 0,82 \\
\hline 8 & 2,94 & 3 & 3 & 1,10 & 0,10 & $-0,66$ & 0,78 & 2,80 & 3 & 3 & 0,83 & $-0,17$ & 0,53 & 0,77 \\
\hline 9 & 2,91 & 3 & 3 & 1,14 & 0,05 & $-0,79$ & 0,84 & 2,72 & 3 & 3 & 0,87 & $-0,12$ & 0,04 & 0,82 \\
\hline 10 & 2,93 & 3 & 2 & 1,28 & 0,08 & $-1,14$ & 0,84 & 2,70 & 3 & 3 & 1,01 & 0,16 & $-0,27$ & 0,77 \\
\hline
\end{tabular}

$\lambda$ : peso fatorial; As: assimetria; Cu: curtose; DP: desvio padrão; Md: mediana; Me: média; Mo: moda.

Tabela 3

Indicadores psicométricos da Escala de Estresse na Alimentação de Salzburg para as amostras de adultos brasileiros.

\begin{tabular}{lcccccccc}
\hline Amostra & $\mathbf{n}$ & CFI & TLI & SRMR & IE & r & VEM & $\mathbf{a}$ \\
\hline Feminina & 634 & 0,95 & 0,93 & 0,06 & - & & 0,63 & 0,94 \\
Masculina & 396 & 0,92 & 0,90 & 0,08 & - & 0,58 & 0,91 \\
Masculina refinado & 396 & 0,94 & 0,92 & 0,07 & 5 & $8-9$ & 0,62 & 0,93 \\
\hline
\end{tabular}

a: coeficiente alfa ordinal; $\lambda$ : peso fatorial; CFI: índice de ajuste comparativo; IE: item excluído; r: correlação entre os erros dos itens; SRMR: raiz quadrada média residual padronizada; TLI: índice de Tucker-Lewis; VEM: variância extraída média. 
feminina (valores de CFI dos modelos: configural $=0,95$; métrico $=0,95$; escalar $=0,96$ ) quanto para a masculina (configural $=0,94$; métrico $=0,94$; escalar $=0,95$ ), os modelos apresentaram invariância forte $(\Delta \mathrm{CFI}<-0,01)$.

Com relação aos modelos estruturais hipotéticos construídos a partir dos modelos fatoriais da SSES ajustados para cada sexo, observou-se que, para ambas as amostras, a idade não exerceu impacto significativo na alimentação frente ao estresse (modelo feminino: $\beta=-0,08, \mathrm{p}=0,05$; modelo masculino: $\beta=-0,67, p=0,19$ ), enquanto que o IMC exerceu (modelo feminino: $\beta=0,27, p<0,01$; modelo masculino: $\beta=0,23, p<0,01$ ), sendo que, quanto maior esse índice, maior foi o consumo de alimentos diante de situações estressantes. Após exclusão da idade, observou-se a manutenção da significância do IMC (modelo feminino: $\beta=0,27, p<0,01$; modelo masculino: $\beta=0,23, \mathrm{p}<0,01$ ), e o ajustamento foi aceitável (modelo feminino: $\mathrm{CFI}=0,95$, $\mathrm{TLI}=0,93$, $\mathrm{SRMR}=0,06$; modelo masculino: $\mathrm{CFI}=0,94$, $\mathrm{TLI}=0,92$, SRMR $=0,07$ ).

O escore médio de alimentação frente ao estresse (i.e., da SSES) para a amostra feminina foi de 2,96 $(\mathrm{DP}=1,14)$ e para a masculina foi de $2,75(\mathrm{DP}=0,73)$. No que se refere à distribuição dos indivíduos nas categorias, observou-se, na amostra masculina, maior frequência na "redução da alimentação habitual frente ao estresse" (54,3\%, IC95\%: 49,8-59,1), quando comparada à amostra feminina (42\%, IC95\%: 38,0-45,4). Já para a "manutenção da alimentação habitual frente ao estresse", não houve diferença entre os sexos (amostra feminina: 18,5\%, IC95\%: 15,5-21,8; amostra masculina: 15,9\%, IC95\%: 12,4-19,7). Por outro lado, na categoria "aumento da alimentação habitual frente ao estresse", a amostra feminina apresentou maior frequência $(39,6 \%$, IC95\%: 35,6-43,4), quando comparada à amostra masculina (29,8\%, IC95\%: 25,5-34,1).

\section{Discussão}

O estresse tem sido uma afetividade prevalente na população mundial. De acordo com a Associação Americana de Psicologia, mais de três quartos dos adultos americanos apresentam sintomas físicos ou emocionais decorrentes do estresse 34 . No Brasil, uma pesquisa representativa da população adulta revelou que mais de 50\% dos indivíduos experienciaram sintomas de estresse 35 . Esses dados apontam o crescimento do estresse, despertando a necessidade de um olhar mais cuidadoso diante das consequências negativas que podem ocorrer na saúde física e mental dos indivíduos. Geralmente, frente ao estresse, os indivíduos tendem a consumir mais alimentos palatáveis e menos alimentos naturais como frutas, hortaliças e vegetais 11. Isso pode contribuir para desfechos como obesidade, alterações bioquímicas/metabólicas 1,7 e distúrbios alimentares 3 . Segundo Sinha \& Jastreboff 7, o estresse diminui o controle emocional e comportamental, aumenta a impulsividade e o consumo de alimentos palatáveis. Além disso, a alta disponibilidade e a exposição a esses alimentos, que têm sido cada dia mais comum, estimulam a via de recompensa cerebral e aumentam a probabilidade de consumo 7. Diante desses fatos, o uso de ferramentas que contribuam para rastrear a alimentação relacionada ao estresse torna-se relevante a fim de desenvolver estratégias para reduzir os desfechos negativos na população. Desse modo, destaca-se a SSES que foi adaptada no presente estudo para a língua portuguesa, visando abrir espaço para identificar o impacto do estresse na alimentação dos indivíduos em contexto brasileiro, que carece de instrumentos nessa temática. Essa versão poderá contribuir tanto com futuros estudos quanto com protocolos clínicos, uma vez que se trata de um instrumento de fácil uso e rápida aplicação. Contudo, vale mencionar que o uso da SSES em todas as regiões do país deve ser cuidadosamente avaliado, uma vez que o processo de alimentação e nutrição pode sofrer influência de aspectos relacionados à cultura, à tradição e a outros fatores.

Quanto ao modelo fatorial da SSES testado para as amostras brasileiras, observou-se que o instrumento avalia a alteração da alimentação frente ao estresse de maneira distinta entre os sexos. Para o sexo masculino, o item cinco ("Enquanto eu me preparo para fazer uma tarefa árdua/cansativa") apresentou baixo peso fatorial e foi excluído. As respostas dadas a esse item indicaram que a realização de tarefas árduas/cansativas talvez não seja extenuante o suficiente para os participantes do sexo masculino a ponto de influenciar na alimentação, diferentemente do que ocorreu para o público feminino. Resultado similar também foi apontado no estudo de desenvolvimento da SSES realizado com amostra de adultos jovens europeus 10 , o que reforça a fragilidade do item cinco para mensurar 
o conceito investigado. Com relação à adequada validade convergente e à boa confiabilidade da SSES para os dados coletados no Brasil, tais achados vão ao encontro do estudo original da escala 10 . No que se refere à diferença do modelo fatorial segundo sexo, esse resultado pode ser sustentado pela literatura que aponta que mulheres e homens apresentam comportamentos distintos com relação à alimentação, à imagem corporal e ao estresse 36,37,38,39. É importante esclarecer que não foi possível realizar comparações diretas dos modelos fatoriais da SSES ajustados para cada sexo com outros trabalhos, por se tratar de um instrumento desenvolvido recentemente e, portanto, as evidências psicométricas ainda são preliminares. Assim, sugerimos que futuros estudos busquem avaliar psicometricamente a SSES em outros contextos/amostras visando comparar seus achados com os resultados do presente trabalho.

Segundo Bennett et al. 40, em estudo realizado com jovens americanos, os resultados indicaram que o estresse afeta de maneira oposta o comportamento alimentar das mulheres e dos homens. De acordo com os autores, as mulheres foram mais propensas a aumentarem o consumo de alimentos, enquanto os homens, mais propensos a reduzir o consumo habitual 40. Esse resultado está em consonância com o observado no presente trabalho, indicando que as mulheres apresentam maior propensão a utilizar a alimentação como forma de enfrentamento, diferente dos homens que tendem a utilizar outras estratégias 18. Já para a manutenção da alimentação frente ao estresse, os resultados não demonstraram diferença entre os sexos. Essa categoria foi a que apresentou menor frequência dos indivíduos, o que corrobora com a literatura, que indica que a maior parte dos indivíduos tendem a alterar a alimentação frente ao estresse, e pequena parcela mantém a alimentação inalterada 12 . Vale reforçar que as categorias utilizadas para classificar os indivíduos de acordo com os escores médios da SSES foram desenvolvidas para o presente estudo e, portanto, devem ser cuidadosamente avaliadas antes do uso em futuros protocolos.

A influência da idade e do IMC na alteração da alimentação frente ao estresse também foi investigada no estudo. No que se refere à idade, não foi observada relação significativa. A ausência de relação entre idade e alimentação frente ao estresse pode ter ocorrido pela delimitação da faixa etária dos indivíduos incluídos no estudo (18 a 35 anos). Abdella et al. ${ }^{21}$ sugerem que os indivíduos mais jovens tendem a comer mais alimentos motivados pelo prazer quando comparados aos mais velhos, contudo, a faixa etária avaliada pelos autores foi de 18 a 81 anos. Esse resultado foi justificado pela menor habilidade das pessoas mais jovens de neutralizar emoções diante de uma situação estressora ${ }^{41}$. Deve-se destacar que, diferente do presente trabalho, o estudo de Abdella et al. 21 investigou outros estados emocionais além do estresse. Já o trabalho realizado por Kandiah et al. 42 , que teve como objetivo avaliar o efeito do estresse no apetite e hábitos alimentares de mulheres jovens americanas, também não encontrou relação entre idade e alimentação, o que corrobora com os achados do presente trabalho. Vale mencionar que a faixa etária avaliada pelos autores foi de 17 a 26 anos 42. Assim, a variabilidade da idade da nossa amostra pode ter sido pequena para detectar diferenças relevantes.

Por outro lado, o IMC apresentou influência significativa na alteração da alimentação frente ao estresse, o que corrobora com o estudo original 10 e outros trabalhos 4,10 encontrados na literatura. Tais pesquisas indicam que, quanto maior o IMC, mais os indivíduos tendem a comer em resposta ao estresse. Popkess-Vawter et al. 13 realizaram estudo com mulheres adultas americanas, e, segundo os autores, as mulheres classificadas segundo o IMC com sobrepeso ou obesidade relataram mais ansiedade, tensão e estresse antes de episódios de comer em excesso, quando comparadas a mulheres com peso normal. O estudo longitudinal realizado por Laitinen et al. 6 com indivíduos adultos europeus, encontrou relação significativa entre IMC e indivíduos que ingeriam alimentos e bebidas motivados pelo estresse. Os autores mencionam a importância do uso de métodos que auxiliem na identificação das formas de enfrentamento do estresse 6 na população. Nesse contexto, o uso da SSES pode ser interessante para avaliar se o indivíduo utiliza a alimentação para lidar com o estresse e, assim, propor estratégias mais assertivas.

O presente trabalho possui algumas limitações. A primeira refere-se à amostra, que foi constituída majoritariamente por indivíduos com renda mensal média alta e peso adequado para a altura (dados que não representam a população brasileira como um todo), e isso, juntamente com o delineamento amostral não probabilístico, limita a inferência dos resultados para outras amostras/contextos com características distintas. Considerando que tanto a prevalência de sintomas de estresse quanto de alimentação desordenada pode diferir de acordo com a classe econômica e o estado nutricional 
antropométrico das pessoas, sugere-se que futuros estudos incluam amostra suficiente para avaliar tais características. A não realização de invariância fatorial em outros grupos, além de subamostras independentes dentro de cada sexo, também pode ser considerada uma limitação do estudo. Além disso, o desenho de estudo transversal não permite estabelecer relação de causa e efeito entre as variáveis investigadas nos modelos estruturais. Ainda, a inclusão de outros instrumentos no protocolo de coleta de dados junto à SSES para investigar as validades concorrente e divergente não foi realizada. Por último, a investigação do consumo alimentar diante de situações estressantes não foi incluída no protocolo de pesquisa. Desse modo, sugerimos que estudos futuros considerem essas limitações para elaborar seus protocolos.

\section{Implicações práticas}

Visto que o crescente aumento das exigências diárias tem desencadeado estresse nos indivíduos e que parte da população tende a utilizar os alimentos para enfrentar as situações estressoras, torna-se relevante ampliar as ferramentas de trabalho dos profissionais da saúde que permitam identificar a alteração da alimentação frente ao estresse. Nesse sentido, espera-se que, ao disponibilizar a versão em português da SSES, bem como suas propriedades psicométricas, pesquisadores e clínicos utilizem essa escala para desenvolver protocolos epidemiológicos ou clínicos visando identificar as alterações alimentares dos indivíduos frente ao estresse. A SSES trata-se de um instrumento curto, o que facilita sua aplicação e, portanto, pode ser útil para nortear a elaboração de estratégias alternativas de enfrentamento de modo a minimizar ou evitar problemas futuros.

\section{Conclusão}

A versão em português da SSES apresentada no presente estudo poderá ser útil para futuros protocolos brasileiros que objetivam investigar a alimentação frente ao estresse. Os dados obtidos com a SSES apresentaram adequada validade e confiabilidade a partir do modelo original para a amostra feminina e de um modelo refinado para a amostra masculina. Isso indica que a operacionalização do instrumento foi distinta entre os sexos, sendo esse resultado importante de ser considerado em avaliações posteriores da escala com amostras similares a do presente trabalho. Ainda, verificou-se que, quanto maior o IMC, maior a alteração da alimentação diante de situações estressoras. Portanto, o estado nutricional antropométrico deve ser investigado quando da avaliação da alimentação frente ao estresse. 


\section{Colaboradores}

P. C. Santos participou da concepção do trabalho, desenho do estudo, contribuiu com a análise e interpretação dos dados e escreveu a primeira versão do manuscrito. W. R. Silva e J. A. D. B. Campos participaram da concepção do trabalho, desenho do estudo, contribuiu com a análise e interpretação dos dados e revisou a versão final do manuscrito para publicação. J. Marôco contribuiu com a análise e interpretação dos dados e revisou a versão final do manuscrito para publicação.

\section{Conflito de interesse}

Os autores não possuem conflitos de interesse a serem declarados.

\section{Informações adicionais}

ORCID: Priscila Carvalho Santos (0000-00017344-2342); Wanderson Roberto da Silva (00000001-8897-8772); João Marôco (0000-0001-92145378 ); Juliana Alvares Duarte Bonini Campos (0000-0001-7123-5585).

\section{Agradecimentos}

À Coordenação de Aperfeiçoamento de Pessoal de Nível Superior (CAPES - código de financiamento 001) e da Fundação de Amparo à Pesquisa do Estado de São Paulo (FAPESP - processo no 2019/195909). À Faculdade de Ciências Farmacêuticas da Universidade Estadual Paulista (UNESP, campus de Araraquara) pelo apoio institucional. A todos os participantes do estudo e aos técnicos de laboratório, Beatriz Buda Fuller e Arthur Fiorin Ragazzi, pelo auxílio na coleta e elaboração do banco de dados.

\section{Referências}

1. Torres SJ, Nowson CA. Relationship between stress, eating behavior, and obesity. Nutrition 2007; 23:887-94.

2. Reichenberger J, Kuppens P, Liedlgruber M, Wilhelm FH, Tiefengrabner M, Ginzinger S, et al. No haste, more taste: an EMA study of the effects of stress, negative and positive emotions on eating behavior. Biol Psychol 2018; 131:54-62.

3. Yau YH, Potenza MN. Stress and eating behaviors. Minerva Endocrinol 2013; 38:255-67.

4. O'Connor DB, Jones F, Conner M, McMillan B, Ferguson E. Effects of daily hassles and eating style on eating behavior. Health Psychol 2008; 27(1S):S20-31.

5. Oliver G, Wardle J, Gibson EL. Stress and food choice: a laboratory study. Psychosom Med 2000; 62:853-65.

6. Laitinen J, Ek E, Sovio U. Stress-related eating and drinking behavior and body mass index and predictors of this behavior. Prev Med 2002; 34:29-39.

7. Sinha R, Jastreboff AM. Stress as a common risk factor for obesity and addiction. Biol Psychiatry 2013; 73:827-35.

8. Adam TC, Epel ES. Stress, eating and the reward system. Physiol Behav 2007; 91:449-58.

9. Singh M. Mood, food, and obesity. Front Psychol 2014; 5:925.

10. Meule A, Reichenberger J, Blechert J. Development and preliminary validation of the Salzburg Stress Eating Scale. Appetite 2018; 120:442-8.

11. Groesz LM, McCoy S, Carl J, Saslow L, Stewart J, Adler N, et al. What is eating you? Stress and the drive to eat. Appetite 2012; 58:717-21.

12. Macht M. How emotions affect eating: a fiveway model. Appetite 2008; 50:1-11.

13. Popkess-Vawter S, Brandau C, Straub J. Triggers of overeating and related intervention strategies for women who weight cycle. Appl Nurs Res 1998; 11:69-76.

14. Ozier AD, Kendrick OW, Knol LL, Leeper JD, Perko M, Burnham J. The Eating and Appraisal Due to Emotions and Stress (EADES) questionnaire: development and validation. J Am Diet Assoc 2007; 107:619-28.

15. Nolan LJ, Halperin LB, Geliebter A. Emotional Appetite Questionnaire. Construct validity and relationship with BMI. Appetite 2010; 54:314-9.

16. Eating Behavior Laboratory, Centre for cognitive neuroscience and Department of Psychology. Salzburg Emotional Eating Scale (SEES). https://www.eat.sbg.ac.at/resources/ salzburg-emotional-eating-scale (acessado em 10/Nov/2020).

17. Marôco J. Análise de equações estruturais: fundamentos teóricos, software e aplicações. 3a Ed. Pêro Pinheiro: ReportNumber; 2021.

18. Jiang Y, King JM, Prinyawiwatkul W. A review of measurement and relationships between food, eating behavior and emotion. Trends Food Sci Technol 2014; 36:15-28. 
19. Greeno CG, Wing RR. Stress-induced eating. Psychol Bull 1994; 115:444-64.

20. Pelchat ML. Food cravings in young and elderly adults. Appetite 1997; 28:103-13.

21. Abdella HM, El Farssi HO, Broom DR. Eating behaviours and food cravings: influence of age, sex, BMI and FTO genotype. Nutrients 2019; 11:377.

22. Hair Jr JF, Black WC, Babin BJ, Anderson RE. Multivariate data analysis. Upper Saddle River: Cengage Learning; 2018.

23. De Onis M, Onyango AW, Borghi E, Siyam A, Nishida C, Siekmann J. Development of a WHO growth reference for school-aged children and adolescents. Bull World Health Organ 2007; 85:660-7.

24. World Health Organization. Obesity: preventing and managing the global epidemic. Geneva: World Health Organization; 2000.

25. Associação Brasileira de Empresas de Pesquisa. Critério de Classificação Econômica Brasil. http://www.abep.org/ (acessado em 01/Set/ 2020).

26. Beaton DE, Bombardier C, Guillemin F, Ferraz MB. Guidelines for the process of cross-cultural adaptation of self-report measures. Spine 2000; 25:3186-91.

27. Hu Lt, Bentler PM. Cutoff criteria for fit indexes in covariance structure analysis: conventional criteria versus new alternatives. Struct Equ Modeling 1999; 6:1-55.

28. Fornell C, Larcker DF. Evaluating structural equation models with unobservable variables and measurement error. J Mark Res 1981; 18:39-50.

29. Pearl J, Bareinboim E. External validity: from do-calculus to transportability across populations. Statist Sci 2014; 29:579-95.

30. Cheung GW, Rensvold RB. Evaluating goodness-of-fit indexes for testing measurement invariance. Struct Equ Modeling 2002; 9:23355.

31. Rossel Y. Lavaan: an R package for structural equation modeling. Version 0.5-12 (BETA). J Stat Softw 2012; 48:1-36.

32. Jorgensen TD, Pornprasertmanit S, Schoemann AM, Rosseel Y. semTools: useful tools for structural equation modeling. https:// CRAN.R-project.org/package $=$ semTools (acessado em 10/Nov/2020).
33. Revelle W. psych: procedures for psychological, psychometric, and personality research. Evanston: Northwestern University Evanston; 2020.

34. American Psychological Association. Stress relief is within reach. https://www.apa.org/top $\mathrm{ics} /$ stress (acessado em 10/Nov/2020).

35. Campos JADB, Martins BG, Campos LA, Marôco J, Saadiq RA, Ruano R. Early psychological impact of the COVID-19 pandemic in Brazil: a national survey. J Clin Med 2020; 9:2976.

36. Peneau S, Menard E, Mejean C, Bellisle F, Hercberg S. Sex and dieting modify the association between emotional eating and weight status. Am J Clin Nutr 2013; 97:1307-13.

37. de Lauzon B, Romon M, Deschamps V, Lafay L, Borys JM, Karlsson J, et al. The Three-Factor Eating Questionnaire-R18 is able to distinguish among different eating patterns in a general population. J Nutr 2004; 134:2372-80.

38. Camilleri GM, Méjean C, Kesse-Guyot E, Andreeva VA, Bellisle F, Hercberg S, et al. The associations between emotional eating and consumption of energy-dense snack foods are modified by sex and depressive symptomatology. J Nutr 2014; 144:12640-73.

39. Silva WR, Swami V, Neves AN, Marôco J, Ochner CN, Campos JADB. The Body Shape Questionnaire is not invariant across sex: evidence from portuguese-speaking university students. Percept Mot Skills 2019; 126:462-76.

40. Bennett J, Greene G, Schwartz-Barcott D. Perceptions of emotional eating behavior. A qualitative study of college students. Appetite 2013; 60:187-92.

41. Charles ST, Carstensen LL. Social and emotional aging. Annu Rev Psychol 2010; 61:383409.

42. Kandiah J, Yake M, Jones J, Meyer M. Stress influences appetite and comfort food preferences in college women. Nutr Res 2006; 26:11823. 


\section{Abstract}

The study's objectives were to adapt the Salzburg Stress Eating Scale (SSES) to Brazilian Portuguese, estimate its psychometric properties in a sample of young adults, and verify (separately for each sex) the relationship between age and BMI and dietary intake during stress. Adaptation of the SSES to Portuguese was performed according to the consolidated protocol. The psychometric properties were estimated for each sex based on factor and convergent validities and reliability. Invariance was tested in independent subsamples for each sex. The relationship between age and bod mass index (BMI) and dietary intake during stress was investigated for each sex using structural equation modeling. Prevalence of individuals in categories that represented maintenance, reduction, or increases in dietary intake in the face of stress was calculated. Participation in the study included 1,030 individuals (61.8\% women) with a mean age of $25.5(S D=5.3)$ years. The original model of the SSES presented good fit for the female sample, but for the male sample one item was excluded and a residual correlation was inserted. These models were invariant in independent subsamples. High BMI influenced dietary intake in the face of stress. Men maintained their habitual diet, while women increased their dietary intake during stress. The Portuguese version of the SSES will be useful for investigating dietary intake during stress in Brazil. Different models of SESS were adjusted for each sex. BMI was a significant variable for assessing dietary intake in the face of stress.

Emotional Distress; Diet; Psychometric; Validation Study

\section{Resumen}

Los objetivos de este estudio fueron adaptar la Escala de Estrés en la Alimentación de Salzburg (SSES) al portugués brasileño, estimar sus propiedades psicométricas en una muestra de adultos jóvenes y verificar, separadamente para cada sexo, la relación entre edad e indice de masa corporal (IMC) en lo que respecta a la alimentación frente al estrés. La adaptación de la SSES al portugués se realizó siguiendo un protocolo consolidado. Las propiedades psicométricas se estimaron para cada sexo a partir de la validez factorial y convergente, así como de la confiabilidad. La invariancia se probó en submuestras independientes para cada sexo. La relación de la edad e IMC con la alimentación frente al estrés se investigó para cada sexo, usando un modelado de ecuaciones estructurales. Se calculó la prevalencia de los individuos dentro de categorías que representaban: mantenimiento, reducción o aumento de la alimentación frente al estrés. Participaron en el estudio 1030 individuos (61,8\% mujeres) con una media de edad de 25,5 $(D P=5,3)$ años. El modelo original de la SSES presentó un buen ajuste en la muestra femenina, sin embargo, en la masculina, se excluyó un ítem y se incluyó una correlación residual. Estos modelos fueron invariantes en submuestras independientes. El IMC elevado influenció en la alimentación frente al estrés. Los hombres mantienen la alimentación habitual, mientras que las mujeres la aumentan frente al estrés. La versión en portugués de la SSES será útil para investigar la alimentación ante el estrés en Brasil. Se ajustaron modelos diferentes de la SESS para cada sexo. El IMC fue una variable significativa para evaluar la alimentación ante el estrés.

Estrés Emocional; Dieta; Psicometría; Estudio de Validación
Recebido em 01/Fev/2021

Versão final reapresentada em 17/Mai/2021

Aprovado em 18/Jun/2021 